\title{
Recapitulation of previous genome-wide association studies with two distinct pathophysiological entities of gastric cancer in the Korean population
}

\author{
Joo-Yeon Hwang ${ }^{1}$, Duk-Hwan Kim ${ }^{2,3}$, Yong-Ick Ji ${ }^{3}$, Min Jin Go ${ }^{1}$, Lyong $\mathrm{Heo}^{1}$, Young Jin $\mathrm{Kim}^{1}$, \\ Tae Sung Sohn ${ }^{4}$, Jae Hyung Noh ${ }^{4}$, Sung Kim ${ }^{4}$, Yeon-Su Lee ${ }^{5}$, Sook-Young Kim ${ }^{5}$, Young-Woo Kim ${ }^{6}$, \\ Keun Won Ryu ${ }^{6}$, Il Ju Choi ${ }^{6}$, Juyoung Lee ${ }^{1}$, Bong-Jo Kim ${ }^{1}$, Bok-Ghee Han ${ }^{1}$, Joobae Park ${ }^{2,3}$ and Jong-Young Lee ${ }^{1}$ \\ Gastric cancer (GC) is the most common malignancy. The incidence rates remain remarkably high in East Asians. Although \\ genome-wide association studies in the Han Chinese and Japanese populations have so far yielded susceptibility loci for GC, \\ these findings need to be validated in an independent ethnic group. To identify the potential heterogeneity by histological \\ classified subtypes (intestinal and diffuse), we examined the previously reported associations in the Korean population. \\ PRKAA1 at 5p13.1 was found to be more strongly associated with intestinal type (odds ratio, $0 \mathrm{R}=1.39,95 \% \mathrm{Cl}$ (confidence \\ interval) $=1.22-1.58, P=3.77 \times 10^{-7}$ ) than diffuse type. In addition, $P S C A$ at $8 q 23.3$ was significantly replicated in diffuse \\ type $\left(\mathrm{OR}=1.49,95 \% \mathrm{Cl}=1.32-1.67, P=2.43 \times 10^{-11}\right)$ but far less significant in intestinal type. In conclusion, these \\ findings could bring additional insights into the etiologic heterogeneity in gastric carcinogenesis mechanisms. \\ Journal of Human Genetics (2013) 58, 233-235; doi:10.1038/jhg.2012.158; published online 7 February 2013
}

Keywords: diffuse; gastric cancer; GWAS; intestinal; Korean

Gastric cancer (GC) is the second leading cause of cancer-related death worldwide. GC consists of two pathological entities (intestinal and diffuse) by histotype-based prognostic classification. ${ }^{1}$ Previous epidemiologic studies have highlighted remarkable geographical or ethnic variations in the age-standardized incidence rates, which is particularly high in East Asia. Korea has the highest incidence and mortality rates for GC in males, followed by Japan and China, respectively. ${ }^{2}$

Gastric carcinogenesis is mainly caused by a combination of environmental risk factors, including Helicobacter pylori infection, dietary habits (high salt/nitrates intake), smoking, alcohol and precancerous conditions. ${ }^{3}$ However, although genetic predisposition is known to have a role in the pathogenesis of GC, its exact mechanism still remains unclear.

Recently, a genome-wide association study (GWAS) of non-cardia GC in the Han Chinese population identified two novel loci at 5p13.1 and 3 p13.31 without any observation in specific histological features or in an independent ethnic group. ${ }^{4}$ In addition, a two-stage GWAS in the Japanese population demonstrated a significant association in PSCA highly related to diffuse type with functional implications in gastric epithelial cell proliferation. ${ }^{5}$
As a useful tool for maximizing information from GWAS, allelespecific DNA methylation is also known to be a promising biomarker for GC risk screening, prediction and prognostication. ${ }^{6}$ Different

Table 1 Clinical characteristics of participants in the Korean population

\begin{tabular}{|c|c|c|c|c|}
\hline \multirow[b]{2}{*}{ Histological subtype } & \multicolumn{2}{|l|}{ Case } & \multicolumn{2}{|c|}{ Control } \\
\hline & No. or mean & $\%$ & No. or mean & $\%$ \\
\hline \multicolumn{5}{|l|}{ Intestinal } \\
\hline Total & 868 & & 1604 & \\
\hline Age $^{a}$ & $60.28 \pm 9.88$ & & $54.65 \pm 8.73$ & \\
\hline Male gender & 692 & 79.7 & 1277 & 79.6 \\
\hline \multicolumn{5}{|l|}{ Diffuse } \\
\hline Total & 890 & & 2189 & \\
\hline Age $^{a}$ & $52.65 \pm 11.99$ & & $53.83 \pm 8.54$ & \\
\hline Male gender & 520 & 58.4 & 1277 & 58.3 \\
\hline
\end{tabular}

aMean \pm s.d.

${ }^{1}$ Center for Genome Science, National Institute of Health, Chungcheongbuk-do, Republic of Korea; ${ }^{2}$ Department of Molecular Cell Biology, Sungkyunkwan University school of Medicine, Suwon, Korea; ${ }^{3}$ Center for Genome Research, Samsung Biomedical Research Institute, Seoul, Korea; ${ }^{4}$ Department of Surgery, Samsung Medical Center and Sungkyunkwan University School of Medicine, Seoul, Korea; ${ }^{5}$ Functional Genomics Branch, National Cancer Center, Goyang, Korea and ${ }^{6}$ Center for Gastric Cancer, National Cancer Center, Goyang, Korea

Correspondence: Dr J-Y Lee, Center for Genome Science, National Institute of Health, Osong Health Technology Administration complex, Chungcheongbuk-do, Chungbuk 363-951, Republic of Korea.

E-mail: leejy63@nih.go.kr

Received 22 August 2012; revised 5 November 2012; accepted 11 December 2012; published online 7 February 2013 
Table 2 The heterogeneity of associations by histotypes in the Korean population

\begin{tabular}{|c|c|c|c|c|c|c|c|c|c|c|c|c|c|c|c|c|}
\hline \multirow[b]{2}{*}{$r s / D$} & \multirow[b]{2}{*}{ Gene } & \multirow[b]{2}{*}{ Chr. } & \multirow{2}{*}{$\begin{array}{c}\text { Risk/non- } \\
\text { risk }\end{array}$} & \multicolumn{6}{|c|}{ Previous GWAS } & \multicolumn{7}{|c|}{ Replication } \\
\hline & & & & Type & Case & Control & $O R$ & $\mathrm{Cl}$ & $P$ & Type & Case & Control & $O R$ & $\mathrm{Cl}$ & $P$ & $H W E^{a}$ \\
\hline \multirow[t]{2}{*}{ rs13361707 } & PRKAA1 & $5 p 13.1$ & $\mathrm{C} / \mathrm{T}$ & $\mathrm{NC}$ & 979 & 2268 & 1.42 & $1.24-1.62$ & $4.3 \times 10^{-7}$ & । & 855 & 1582 & 1.39 & $1.22-1.58$ & $3.77 \times 10^{-7}$ & $1.29 \times 10^{-1}$ \\
\hline & & & & & & & & & & D & 881 & 2164 & 1.2 & $1.07-1.34$ & $1.34 \times 10^{-3}$ & $6.59 \times 10^{-1}$ \\
\hline \multirow[t]{2}{*}{ rs2976392 } & $P S C A$ & $8 q 24.3$ & $A / G$ & I & 599 & 1397 & 1.29 & $1.12-1.49$ & $5.0 \times 10^{-4}$ & । & 861 & 1574 & 1.2 & $1.06-1.36$ & $5.25 \times 10^{-5}$ & $7.30 \times 10^{-2}$ \\
\hline & & & & D & 926 & 1397 & 1.71 & $1.50-1.94$ & $1.5 \times 10^{-16}$ & D & 875 & 2144 & 1.49 & $1.32-1.67$ & $2.43 \times 10^{-11}$ & $6.33 \times 10^{-1}$ \\
\hline
\end{tabular}

Abbreviations: chr, chromosome; $\mathrm{Cl}$, confidence interval; D, diffuse; HWE, Hardy-Weinberg equilibrium; I, intestinal; NC, non-cardia; OR, odds ratio.

aHWE in controls.

DNA methylation patterns occur by distinct pathways, according to the histological subtypes of gastric adenocarcinomas. ${ }^{7}$ Considering heterogeneous pathogenesis by different subtypes, these findings herein need to be validated for intestinal- and diffuse-type GC in an independent ethnic population.

To clarify the genetic susceptibility to the intestinal- and diffusetype GC in the Korean population, we conducted a replication study for six previous genome-wide associations extracted from Affymetrix Genome-Wide Human SNP (single-nucleotide polymorphism) arrays 6.0 (Affymetrix, Santa Clara, CA, USA) with the intestinal type in 868 cases and 1604 gender-matched controls and the diffuse type in 890 cases and 2189 gender-matched controls from the Samsung Medical Center and the Korean Genome Epidemiology Study. This study was approved by the Institutional Review Board of the Korea National Institute of Health, and each participant provided written informed consent for participation. The basic characteristics of participants are described in Table 1.

To determine etiological influence for novel loci associated with non-cardia GC as a predisposing factor in the Chinese population, we performed an association analysis under an additive model with adjustment for age and gender. The heterogeneous association results in intestinal- and diffuse-type sets are shown in Table 2.

Compared with diffuse-type (odds ratio, OR $=1.2,95 \% \mathrm{CI}$ (confidence interval) $\left.=1.07-1.34, P=1.34 \times 10^{-3}\right)$, rs1336107 in PRKAA1 at 5 p13.1 was significantly associated with an increased risk of intestinaltype $\mathrm{GC}\left(\mathrm{OR}=1.39,95 \% \mathrm{CI}=1.22-1.58, P=3.77 \times 10^{-7}\right)$. PRKAA1 encodes alpha 1 catalytic subunit of the $5^{\prime}$-AMP-activated protein kinase (AMPK), an important energy sensor protein kinase regulating cellular energy metabolism. In response to the stimuli of increased AMP/ATP ratio, AMPK activates catabolic pathways and inhibits ATP-consuming biosynthetic pathways through phosphorylation in cell growth and proliferation. AMPK has been implicated in endocrine-related cancers including gastrointestinal polyps and other epithelial malignancies. ${ }^{8,9}$ Therefore, we suggest that PRKAA1 variant (rs1336107) was identified as a susceptibility locus to intestinal-type gastric carcinogenesis in the Korean population.

However, other loci at 1q22 (rs4072037), 3q13.31 (rs9841504), $10 \mathrm{q} 23$ (rs2274223) and 20p13 (rs13042395), associated with risk of mixed types of GC, were not replicated with significant associations specific for two subtypes in the Korean population, which may be due to the difference in the distribution of risk allele frequencies. ${ }^{10}$ These observations need to be further validated in a large-scale consortium of many ethnic groups, according to ethnicity, histological subtypes, tumor sites and source of controls.

To consolidate phenotype-specific effects, we further investigated the potential association between diffuse-type GC and an intronic SNP (rs2976392) in PSCA. The SNP rs2976392 in PSCA at 8q23.3 was consistently replicated in diffuse type $(\mathrm{OR}=1.49,95 \%$
$\left.\mathrm{CI}=1.32-1.67, P=2.43 \times 10^{-11}\right)$ but far less significant in intestinal-type $\left(\mathrm{OR}=1.2,95 \% \mathrm{CI}=1.06-1.36, \quad P=5.25 \times 10^{-5}\right)$. Our lookup validation revealed specific genetic effects for diffuse-type GC in the same direction as reported in previous GWAS in the Japanese population. ${ }^{5}$ In addition, a meta-analysis by clinicopathological classification found a significantly increased risk of PSCA rs2976392 in non-cardia or diffused type GC. ${ }^{11}$ These results may provide supporting evidence of the shared genetic susceptibility to the histotype-specific feature and prognosis of GC among the Japanese, Chinese and Korean populations. PSCA, expressed in epithelial cell, encodes a glycosylphosphatidylinositol-anchored cell membrane glycoprotein. When considering the functional implications of PSCA in the cell proliferation inhibition and cell death induction, it is plausible that PSCA variants may functionally contribute to increased risk with diffuse-type GC in the carcinogenic process.

In this study, the recapitulation of the heterogeneity for previous associations might strengthen, understanding the etiological pathogenesis of different GC subtypes in East Asian populations. Considering the heterogeneous complexity of GC in genetic and etiological aspects, further molecular classification and geneenvironment association studies are required for defining the genetic architecture of gastric carcinogenesis.

\section{CONFLICT OF INTEREST}

The authors declare no conflict of interest.

\section{ACKNOWLEDGEMENTS}

This work was supported by grants from the Korea Centers for Disease Control and Prevention (4845-301), an intramural grant from the Korea National Institute of Health (2011-N73007-00).

1 Yoshida, T., Ono, H., Kuchiba, A., Saeki, N. \& Sakamoto, H. Genome-wide germline analyses on cancer susceptibility and GeMDBJ database: gastric cancer as an example. Cancer Sci. 101, 1582-1589 (2010).

2 Kamangar, F., Dores, G. M. \& Anderson, W. F. Patterns of cancer incidence, mortality, and prevalence across five continents: defining priorities to reduce cancer disparities in different geographic regions of the world. J. Clin. Oncol. 24, 2137-2150 (2006).

3 Nagini, S. Carcinoma of the stomach: a review of epidemiology, pathogenesis, molecular genetics and chemoprevention. World J. Gastrointest. Oncol. 4, 156-169 (2012).

4 Shi, Y., Hu, Z., Wu, C., Dai, J., Li, H., Dong, J. et al. A genome-wide association study identifies new susceptibility loci for non-cardia gastric cancer at 3q13.31 and 5p13.1. Nat. Genet. 43, 1215-1218 (2011).

5 Sakamoto, H., Yoshimura, K., Saeki, N., Katai, H., Shimoda, T., Matsuno, Y. et al. Genetic variation in PSCA is associated with susceptibility to diffuse-type gastric cancer. Nat. Genet. 40, 730-740 (2008).

6 Sapari, N. S., Loh, M., Vaithilingam, A. \& Soong, R. Clinical potential of DNA methylation in gastric cancer: a meta-analysis. PLoS One 7, e36275 (2012).

7 Alves, M. K., Ferrasi, A. C., Lima, V. P., Ferreira, M. V., de Moura Campos Pardini, M. I. \& Rabenhorst, S. H. Inactivation of COX-2, HMLH1 and CDKN2A gene by promoter 
methylation in gastric cancer: relationship with histological subtype, tumor location and Helicobacter pylori genotype. Pathobiology 78, 266-276 (2011).

8 Brown, K. A., Samarajeewa, N. U. \& Simpson, E. R. Endocrine-related cancers and the role of AMPK. Mol. Cell Endocrinol. (e-pub ahead of print 16 July 2012).

9 Shackelford, D. B. \& Shaw, R. J. The LKB1-AMPK pathway: metabolism and growth control in tumour suppression. Nat. Rev. Cancer 9, 563-575 (2009).
10 Zhang, H., Jin, G., Li, H., Ren, C., Ding, Y., Zhang, Q. et al. Genetic variants at 1q22 and 10 q23 reproducibly associated with gastric cancer susceptibility in a Chinese population. Carcinogenesis 32, 848-852 (2011).

11 Shi, D., Wang, S., Gu, D., Wu, D., Wang, M., Chu, H. et al. The PSCA polymorphisms derived from genome-wide association study are associated with risk of gastric cancer: a meta-analysis. J. Cancer Res. Clin. Oncol. 138, 1339-1345 (2012). 